\title{
Does 2-FDG PET Accurately Reflect Quantitative In Vivo Glucose Utilization?
}

\author{
Jorge R. Barrio ${ }^{1}$, Sung-Cheng Huang ${ }^{1}$, Nagichettiar Satyamurthy ${ }^{1}$, Claudio S. Scafoglio ${ }^{2}$, Amy S. Yu ${ }^{1}$, Abass Alavi ${ }^{3}$, \\ and Kenneth A. Krohn ${ }^{4}$ \\ ${ }^{I}$ Department of Molecular and Medical Pharmacology, David Geffen UCLA School of Medicine, Los Angeles, California; \\ ${ }^{2}$ Department of Medicine, David Geffen UCLA School of Medicine, Los Angeles, California; ${ }^{3}$ University of Pennsylvania, \\ Philadelphia, Pennsylvania; and ${ }^{4}$ Oregon Health and Science University, Portland, Oregon
}

2-Deoxy-2-18 F-fluoro-D-glucose (2-FDG) with PET is undeniably useful in the clinic, being able, among other uses, to monitor change over time using the 2-FDG SUV metric. This report suggests some potentially serious caveats for this and related roles for 2-FDG PET. Most critical is the assumption that there is an exact proportionality between glucose metabolism and 2-FDG metabolism, called the lumped constant, or LC. This report describes that LC is not constant for a specific tissue and may be variable before and after disease treatment. The purpose of this work is not to deny the clinical value of 2-FDG PET; it is a reminder that when one extends the use of an appropriately qualified imaging method, new observations may arise and further validation would be necessary. The current understanding of glucose-based energetics in vivo is based on the quantification of glucose metabolic rates with 2-FDG PET, a method that permits the noninvasive assessment of various human disorders. However, 2-FDG is a good substrate only for facilitatedglucose transporters (GLUTs), not for sodium-dependent glucose cotransporters (SGLTs), which have recently been shown to be distributed in multiple human tissues. Thus, the GLUT-mediated in vivo glucose utilization measured by 2-FDG PET would be masked to the potentially substantial role of functional SGLTs in glucose transport and use. Therefore, under these circumstances, the 2-FDG LC used to quantify in vivo glucose utilization should not be expected to remain constant. 2-FDG LC variations have been especially significant in tumors, particularly at different stages of cancer development, affecting the accuracy of quantitative glucose measures and potentially limiting the prognostic value of 2-FDG, as well as its accuracy in monitoring treatments. SGLT-mediated glucose transport can be estimated using a-methyl-4-deoxy-4-18 F-fluoro-D-glucopyranoside (Me-4FDG). Using both 2-FDG and Me-4FDG should provide a more complete picture of glucose utilization via both GLUT and SGLT transporters in health and disease states. Given the widespread use of 2-FDG PET to infer glucose metabolism, it is relevant to appreciate the potential limitations of 2-FDG as a surrogate for glucose metabolic rate and the potential reasons for variability in LC. Even when the readout for the 2-FDG PET study is only an SUV parameter, variability in LC is important, particularly if it changes over the course of disease progression (e.g., an evolving tumor).

\footnotetext{
Received Sep. 27, 2019; revision accepted Oct. 22, 2019.

For correspondence or reprints contact either of the following: Jorge $R$. Barrio, UCLA School of Medicine, 10833 Le Conte Ave., Los Angeles, CA 90095-6948.

E-mail: jbarrio@mednet.ucla.edu

Kenneth A. Krohn, Oregon Health and Science University, Portland, OR 97239

E-mail: krohke@ohsu.edu

Published online Nov. 1, 2019.

COPYRIGHT (C 2020 by the Society of Nuclear Medicine and Molecular Imaging.
}

Key Words: FDG PET; SGLTs; lumped constant

J Nucl Med 2020; 61:931-937

DOI: 10.2967/jnumed.119.237446

$\mathbf{T}$ he introduction of PET to medical research in the 1970s permitted for the first time the visualization of key biochemical process in living humans, providing a unique tool for the evaluation of the biologic basis of disease $(1,2)$. Since biochemical alterations precede anatomic abnormalities in most diseases, PET was quickly perceived as a unique opportunity for earlier diagnosis before the onset of clinical symptoms, a critical consideration for effective treatments.

The ultimate objective for the use of molecular imaging probes with PET is the quantification of specific biochemical processes. Because the need for energy is ubiquitous in cells, a generally applicable method for measuring energy production was sought. This is highlighted in the use of 2-FDG for the measurement of local glucose metabolism $\left(\mathrm{MR}_{\mathrm{Glc}}\right)$, which is the cornerstone of PET imaging in humans. Glucose is the primary substrate for energy metabolism in tissues, and a continuous supply of glucose is required for cells to function. Thus, glucose utilization measured using 2-FDG PET has become an established method for quantifying in vivo local functional activity in brain, heart, and most cancers.

Because of the design and characteristics of early PET scanners, the first human studies used 2-FDG PET primarily to investigate epilepsy, stroke, and Alzheimer disease and to grade primary brain tumors (3). Mainly for these reasons, most 2-FDG validation studies in tumors were applied to primary brain tumors.

\section{THE SOKOLOFF METHOD FOR IN VIVO GLUCOSE UTILIZATION}

The methodology for using 2-FDG in human imaging was developed from the ${ }^{14} \mathrm{C}$-2-deoxy-D-glucose (2-DG) autoradiography approach of Sokoloff et al. (2) for the measurement of cerebral $\mathrm{MR}_{\mathrm{Glc}}$ in vivo in the normal rat brain. The principle is as follows: the energyindependent facilitated glucose transporters (GLUTs), whose isoform GLUT1 is expressed in the blood-brain barrier (BBB) and glial cells and GLUT3 in neurons (4), have long been considered the main transporters of glucose. After transport, intracellular hexokinase catalyzes the phosphorylation of glucose to glucose-6-phosphate in the first step of glycolysis. This key phosphorylation step by hexokinase was exploited in the 2-DG autoradiography approach 
(2) based on the fact that phosphorylated 2-DG is not metabolized further and is trapped in the cell. The approach has been quantitatively validated, and inference of the glucose metabolic rate from 2-FDG PET relies on the use of a term called the lumped constant (LC) to account for differences in the kinetics of transport and phosphorylation of 2-DG or 2-FDG relative to glucose:

$$
\mathrm{LC}=\frac{\lambda \cdot \mathrm{K}_{\mathrm{m}} \cdot \mathrm{V}_{\max }^{*}}{\phi \cdot \mathrm{V}_{\max } \cdot \mathrm{K}_{\mathrm{m}}^{*}}
$$

where $\lambda$ is the ratio of the distribution volume of $2-\mathrm{DG}$ or $2-\mathrm{FDG}$ to that of glucose and $\phi$ is the fraction of glucose that continues down the Embden-Meyerhof pathway after being phosphorylated, typically close to $1 . \mathrm{K}_{\mathrm{m}}$ is the Michaelis-Menten constant for phosphorylation of glucose (* indicates 2-DG or 2-FDG), and $\mathrm{V}_{\max }$ is the maximum velocity for phosphorylation of glucose $(*$ indicates 2-DG or 2-FDG). The LC is used to convert the metabolic rate of 2-DG $\left(\mathrm{MR}_{2-\mathrm{DG}}\right)$ to that of glucose $\left(\mathrm{MR}_{\mathrm{Glc}}\right)$ by dividing $\mathrm{MR}_{2-\mathrm{DG}}$ by LC:

$$
\mathrm{MR}_{\mathrm{Glc}}=\frac{\mathrm{MR}_{2-\mathrm{DG}}}{\mathrm{LC}} .
$$

It is important to appreciate the assumptions of this model when using it to interpret a 2-FDG PET study. The 2-DG approach assumed that the GLUT pathway was the sole entry point for glucose utilization. Except for some regional variation (i.e., Purkinje cells, glutamatergic neurons), this assumption is essentially true in the healthy brain as demonstrated by Sokoloff's initial validation studies (2) and by our own measurements presented in this article. But there are at least 2 functional transporter systems in humans, GLUTs and sodium-dependent glucose cotransporters (SGLTs), that are open and variable in tissue, and this observation is most prevalent with evolving tumors. Since 2-FDG is masked to SGLT-mediated GLUTs (Table 1), the 2-transporter mechanism has the potential to lead to misinterpretation of cancer diagnosis and staging, particularly at the earliest stages of disease, and also to inaccurate evaluation of treatment response with longitudinal studies. Thus, 2-FDG validation is not nearly as complete for disease conditions as it is for the healthy normal brain. If we are to have confidence in diagnostic or repeat 2-FDG PET studies (e.g., baseline and after treatment), it behooves the nuclear medicine community to invest in more thorough validation research for reasons presented in this report.

\section{VARIABILITY OF THE LC}

The approach pioneered by Sokoloff (2) with 2-DG and autoradiography in normal rat brain was extended to the human brain and first applied using 2-FDG and the Mark IV scanner at the University of Pennsylvania in August 1976 (5). It has since been incorporated worldwide as the 2-FDG PET methodology (6). The 2-FDG PET method assumes that 2-FDG kinetics for transport and phosphorylation and glucose metabolism change in parallel, thereby justifying the LC as a proportionality constant. Although that assumption is valid in healthy brain, global variability of the 2-FDG LC in a variety of pathophysiologic conditions and even in many normal tissues of the body indicates that this assumption is not always correct. For example, using the relative $\mathrm{MR}_{2-\mathrm{DG}}$ and $\mathrm{MR}_{\mathrm{Glc}}$ based on arteriovenous differences across the rat brain, Sokoloff et al. (2) obtained a value for the 2-DG LC of 0.483 (SD, 0.107). Reivich et al. (7), by a similar approach in humans, estimated the LC for 2-FDG to be 0.52 (SD, 0.028). Phelps et al. (6), using dynamic PET imaging of 2-FDG, estimated the LC for 2-FDG to be 0.42 ( $\mathrm{SD}, 0.059$ ). Whereas the direct method introduced by Sokoloff had produced rather stable 2-FDG LC values for the normal human brain, other indirect methods have established the 2-FDG LC to be higher. Graham et al. (8) used kinetic analysis of sequential 2-FDG and $1-{ }^{11} \mathrm{C}$-glucose PET studies to independently measure $\mathrm{MR}_{\mathrm{Glc}}$ and $\mathrm{MR}_{2 \mathrm{FDG}}$ and obtain a uniform 2-FDG LC throughout the healthy brain of 0.89 (SD, 0.08). Other groups have obtained similar values in the healthy human brain: 0.81 (SD, 0.15 ) from one group (9) and $0.81(\mathrm{SD}, 0.18)$ from another (10). Other methods to assess the stability of the 2-FDG LC under a variety of conditions have been proposed, including kinetic constant assessments to estimate glucose transport across the BBB and derive glucose utilization in brain tissue (11).

However, measurement of $\mathrm{MR}_{\mathrm{Glc}}$ and $\mathrm{MR}_{2 \mathrm{FDG}}$ in patients with primary brain tumors resulted in LCs of $1.23 \pm 0.35$ in 10 subjects with anaplastic astrocytoma, $1.47 \pm 0.48$ in 30 subjects with glioblastoma, and $0.86 \pm 0.14$ in contralateral brain of these same subjects (12). The most striking aspect of these reports is that the

TABLE 1

\begin{tabular}{|c|c|c|c|c|c|c|c|c|c|}
\hline \multirow[b]{2}{*}{ Hexose } & \multicolumn{3}{|c|}{ SGLT1/SGLT2 } & \multicolumn{3}{|c|}{ GLUT1 } & \multicolumn{3}{|c|}{ Hexokinase } \\
\hline & $\mathrm{K}_{\mathrm{m}}(\mathrm{mM})$ & Turnover $\left(\mathrm{s}^{-1}\right)$ & Reference & $\mathrm{K}_{\mathrm{m}}(\mathrm{mM})$ & Turnover $\left(\mathrm{s}^{-1}\right)$ & Reference & $\mathrm{K}_{\mathrm{m}}(\mathrm{mM})$ & $\mathrm{V}_{\max }$ & Reference \\
\hline 2-FDG & $>100$ & u.d. & 22 & 3.2 & $1,000-13,000^{*}$ & 39 & 0.19 & 0.5 & 41 \\
\hline 4-FDG & 0.07 & $160^{\dagger}$ & 22 & n.a. & $1,000-13,000^{*}$ & & 84 & 0.1 & 41 \\
\hline Me-4FDG & $0.1^{\ddagger}$ & $160^{\dagger}$ & & u.d. & $\sim 0$ & 25 & u.d. & u.d. & 25 \\
\hline Me-glucose & $0.7 / 1.6$ & 160 & $22,37,38$ & u.d & $\sim 0$ & 39 & u.d. & u.d. & 42 \\
\hline D-glucose & $0.5 / 1.6$ & 160 & $16,37,38$ & 6.3 & $1,000-13,000$ & 39,40 & 0.17 & 1 & 41 \\
\hline
\end{tabular}

Kinetics of Hexose Membrane Transport and Phosphorylation (Adapted from (25))

${ }^{\star}$ Estimated to be identical to $\mathrm{D}$-glucose at $38^{\circ} \mathrm{C}$.

${ }^{\dagger}$ Estimated to be identical to Me-glc (a-methyl-D-glucopyranosides) at $38^{\circ} \mathrm{C}$.

‡Mean of 2 experiments.

Me-DG = 1-methyl-D-glucopyranoside; u.d. = undetectable; n.a. = not available.

On SGLT1/SGLt2, turnover data are only for SGLT1. 


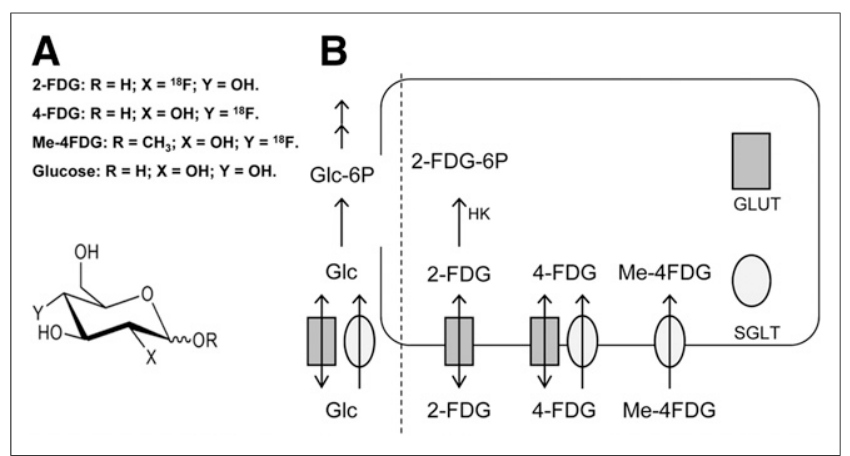

FIGURE 1. GLUT imaging probes and their transport cascades into cells. (A) Structure of glucose analog probes. (B) Graphical interpretation of glucose utilization via GLUT and SGLT: glucose (Glc) is transported into cells by both GLUT and SGLT. To discriminate between various glucose transport pathways, probes were designed to be substrates for GLUT (2-FDG), SGLT (Me-4FDG), or both (4-FDG). 2-FDG is phosphorylated by hexokinase but cannot be further metabolized, resulting in metabolic trapping in cells. Neither 4-FDG nor Me-4FDG is a substrate for hexokinase, consistent with their retention in cells by sodium/glucose cotransport (SGLT) mechanism. (Adapted from (25).)

between-subject variance was much larger for tumors than for normal brain, suggesting that it is inappropriate to extend the assumptions from the Sokoloff model to tumors in the brain.

Extending the use of 2-FDG PET outside the normal brain and brain tumors to quantify glucose metabolism has been equally problematic because of limited validation. In traumatic brain injury, a significant reduction in the global and regional 2-FDG LC was observed and attributed to reduced hexokinase activity $(13,14)$. Beyond the normal brain, no consistent 2-FDG LC could be demonstrated in normal rabbit myocardium (15), human skeletal muscle (16), or adipose tissue (17) —all tissues in which insulin dependency is important. The LC has also been measured in pig liver under fasting and hyperinsulinemia, with substantially different values (18).

\section{PROPOSED REASONS FOR VARIABILITY OF THE LC: THE SGLT EFFECT}

Although the 2-FDG LC in the normal brain has been found to be reasonably stable, publications $(11,19-21)$ have consistently demonstrated that tumors are particularly sensitive to variations in 2-FDG LC. This was attributed to the specific differences between brain tumors and healthy brain, such as tissue heterogeneity in expression of hexokinases, lack of BBB integrity, and regional differences in glucose concentration $(19,21)$. This variability may not be surprising in view of the dysregulated enzymology of tumors.

The properties of hexokinases as well as GLUTs contribute to the net values for $\mathrm{K}_{\mathrm{m}}$ and $\mathrm{V}_{\max }$ in the $\mathrm{LC}$, with phosphorylation considered more rate-limiting than transport. Hexokinase isozymes often have different properties and a subcellular localization (attached to mitochondrial membrane rather than cytosolic) in pathologic tissues. This property has been suggested earlier as a possible explanation for LC variability $(19,21)$. Although it is likely that hexokinase isoenzymes lead to some of the variability in LCs in human tissues, recent observations on the functional role of SGLTs offer a compelling additional explanation for the variation in 2-FDG LC.

SGLT transporters derive from the SLC5A gene family. They harness the gradient of sodium ions across the plasma membrane to drive glucose into cells, using a cotransporter mechanism coupling the movement of 1 glucose and 2 sodium ions (22-24). The most studied family members are SGLT1 and SGLT2, which are mainly involved in glucose transport in the intestine and kidneys to conserve this important substrate for energy metabolism. Even though there has been evidence for SGLT messenger RNA and protein in multiple human tissues (22-24), the full extent of functional SGLTs remained largely unknown until recently $(25,26)$. Indeed, SGLT transporters are widely distributed throughout the body, including in cancer, making it tempting to speculate a priori that their participation in in vivo glucose utilization could be potentially significant (27). This adds a previously unrecognized factor that may critically influence the 2-FDG LC and the accuracy of 2-FDG for quantifying regional $\mathrm{MR}_{\mathrm{Glc}}$. As stated earlier, because 2-FDG is not transported by SGLTs (25), any component of glucose utilization via SGLT transporters (Fig. 1) would be overlooked with 2-FDG, resulting in an underestimation of glucose utilization and an artificial variation in its $L C$. As a result, the regional and global $\mathrm{MR}_{\mathrm{Glc}}$ values determined with 2-FDG PET will not be accurate in an unknown but potentially substantial number of cases (e.g., in tumors). Unfortunately, the literature does not provide measurements of LC in some of the tissues where SGLTs have been shown to be prevalent, such as prostate, pancreas, and lung adenocarcinoma. However, it is safe to expect that human cancers and pathologic states in general in many organs involve significant variability in the 2-FDG LC (19). Despite these limitations, the semiquantitative use of 2-FDG PET has remained useful clinically for diagnosis and to evaluate the prognosis for patients, especially when reported as SUVs.

\section{IMAGING AGENTS FOR SGLT-MEDIATED GLUTS}

SGLT-mediated glucose transport can be imaged with PET in animals and humans. SGLT-specific radiolabeled sugar analogs of 1- $\alpha$-methyl-D-glucopyranoside have been developed as substrates for in vivo assessment of glucose transport using PET (Table 1).

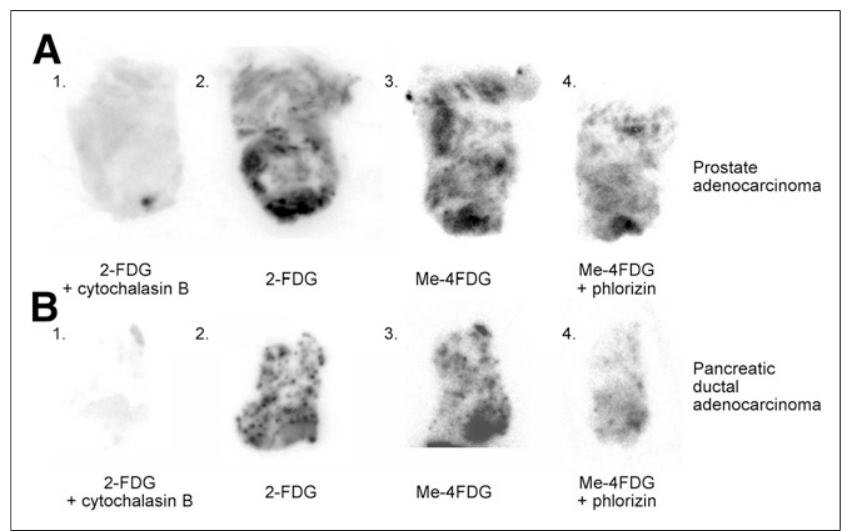

FIGURE 2. Spatial heterogeneity of glucose uptake in pancreatic and prostate cancers in humans. Regional distribution of glucose uptake via GLUTs and SGLTs was mapped in fresh samples of human prostate adenocarcinoma (A) and of pancreatic ductal adenocarcinoma (B) by in vitro uptake and autoradiography. Specimens were cut into $300-\mu \mathrm{m}-$ thick slices, and consecutive slices were incubated with either 2-FDG (specific for GLUTs) or Me-4FDG (which detects SGLT activity), with or without coincubation with corresponding inhibitors (cytochalasin A for GLUT and phlorizin for SGLT). Slices were subsequently exposed to autoradiographic plates to obtain images of regional tracer uptake. Numbers at top left corner of each slice represent order in which slices were cut. (Adapted from (27).) 


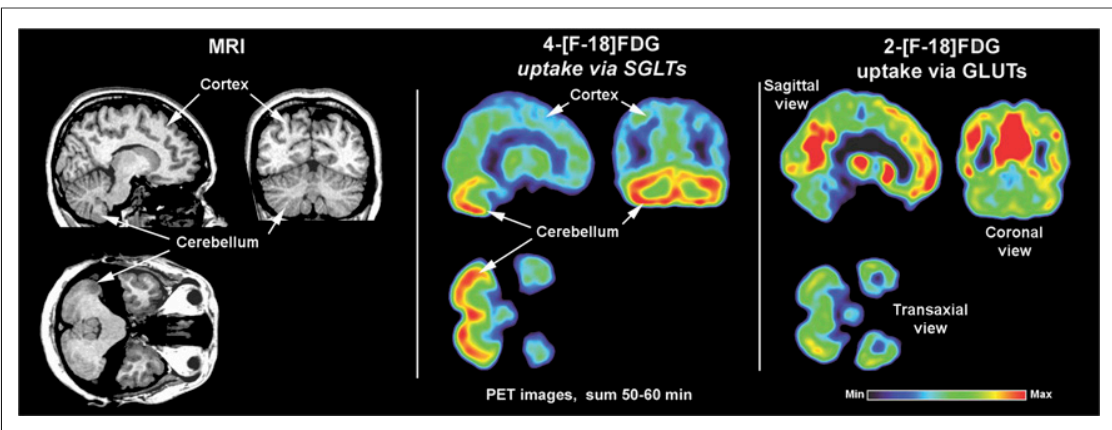

FIGURE 3. SGLT-mediated glucose utilization in normal human brain. The 2 PET panels display typical findings in normal human brain, reflecting GLUT-mediated glucose utilization (right panel) and functional SGLT distribution (left panel) (both images are sum from 50 to 60 min after intravenous injection). Structural MRI scans provide brain anatomic reference. Substantial relative regional differences are seen between the 2 PET scans, most particularly high functional SGLT localization in Purkinje cells in cerebellum, as independently verified by immunohistochemistry. (Courtesy of Vladimir Kepe, Ernest M. Wright, and Jorge R. Barrio.)

Mapping of the distribution of SGLT expression and function is possible with $\alpha$-methyl-4-deoxy-4- ${ }^{18} \mathrm{~F}$-fluoro-D-glucopyranoside (Me4FDG), a SGLT-specific molecular imaging probe with desirable imaging characteristics for quantitative assessment of glucose metabolism via the SGLT pathway (e.g., high affinity, selective specificity,

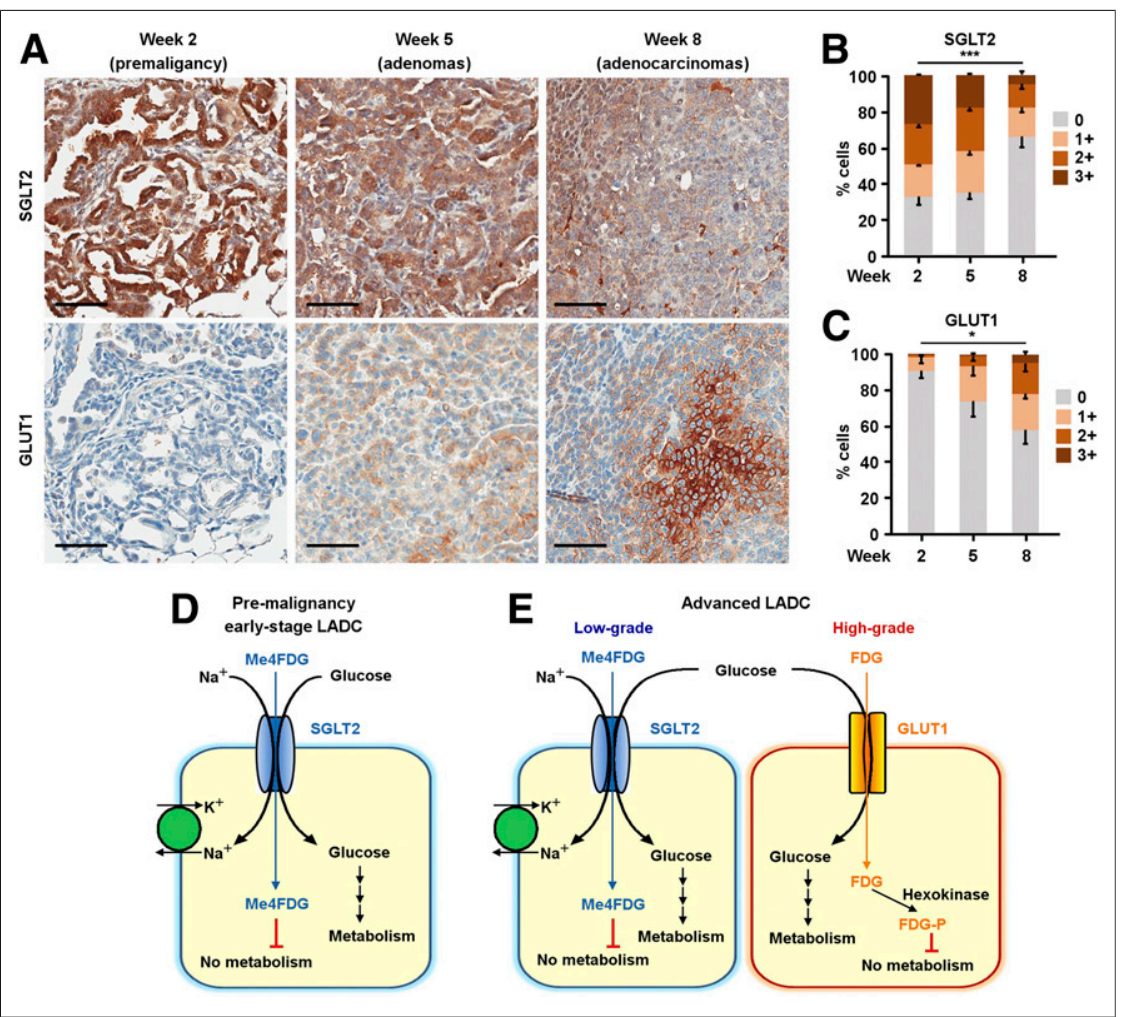

FIGURE 4. Temporal heterogeneity of GLUT expression in lung adenocarcinoma. (A) SGLT2 and GLUT1 expression as investigated in $\mathrm{Kras}^{\mathrm{G} 12 \mathrm{D}}$; p53-/- (KP) model of lung adenocarcinoma at different time points after tumor induction. Scale bar $=50 \mu \mathrm{m}$. (B and C) Expression of SGLT2 (B) and GLUT1 (C) immunohistochemistry signal at different time points after tumor induction. (D and E) Schematic of glucose transporter expression in different stages of lung adenocarcinoma (LADC): premalignant lesions and early-stage lung adenocarcinoma express only SGLT2 (D), whereas advanced tumors show spatial heterogeneity of glucose transport expression, with SGLT2 in well-differentiated areas and GLUT1 in poorly differentiated areas of same tumor (E). Similar spatial heterogeneity of GLUT expression was also demonstrated in human lung adenocarcinoma. (Reprinted with permission of (30).) and no significant peripheral or tissue metabolism) (28). High-affinity Me-4FDG trapping in the SGLT transporter is in contrast to the tissue accumulation of 2-FDG through conversion to 2-FDG-6-phosphate.

Using this approach, the expression of SGLT2 was demonstrated by immunohistochemistry in human astrocytomas, pancreatic cancers, and prostate cancers. Their functional activity was also shown by transport assays on fresh surgical specimens with the SGLT-specific Me-4FDG. Imaging pancreatic and prostate adenocarcinomas using Me-4FDG PET was demonstrated in mouse xenograft models (27) and preliminarily in humans (Ernest M. Wright and Jorge R. Barrio, unpublished data, 2014). Highgrade astrocytomas were also imaged with Me-4FDG PET in humans (29). Furthermore, the importance of SGLT2 in tumor growth and survival in a mouse xenograft model of pancreatic cancer treated with specific SGLT2 inhibitors that are used for treating diabetes has been reported (27). These tumors were highly heterogeneous by immunohistochemistry, with cell aggregates accumulating Me-4FDG as well as 2-FDG (Fig. 2).

For measurement of SGLT function in brain, the molecular imaging probe must also be a good substrate for GLUT1, the exclusive transporter of D-glucose and ana$\operatorname{logs}$ across the BBB. SGLT-specific radiolabeled imaging probes without GLUT1 affinity, such as Me-4FDG, cannot be used for in vivo assessment of SGLT in the human brain since the SGLT transporters are expressed primarily on cortical and hippocampus pyramidal neurons, as well as on Purkinje cells in the cerebellum (Fig. 3) (25). However, Me-4FDG can be used when the $\mathrm{BBB}$ is compromised, as in high-grade astrocytomas, and its specific retention supported by SGLT2 expression in neoplastic glioblastoma cells and endothelial cells of the proliferating microvasculature (29). On the basis of earlier work on the in vitro transport of sugar-analog probes via SGLT1, 4deoxy-4- ${ }^{18}$ F-fluoro-D-glucose (4-FDG) was identified as a suitable biomarker for probing SGLTs in the intact brain $(25,26)$. 4-FDG is qualified for in vivo imaging in that it is an excellent substrate for SGLT transporters, with specific cell retention in proportion to functional activity, while maintaining its reversible transport via GLUT1 at the BBB (Table 1; Fig. 1).

\section{THE SHIFT TO OXIDATIVE GLYCOLYSIS WITH CANCER PROGRESSION: EFFECT ON LC AND SUV}

Tumor heterogeneity can change as cancers develop, further complicating interpretation of 2-FDG PET quantitative determinations, whether by $\mathrm{MR}_{\mathrm{Glc}}$ or by SUV. The shift 


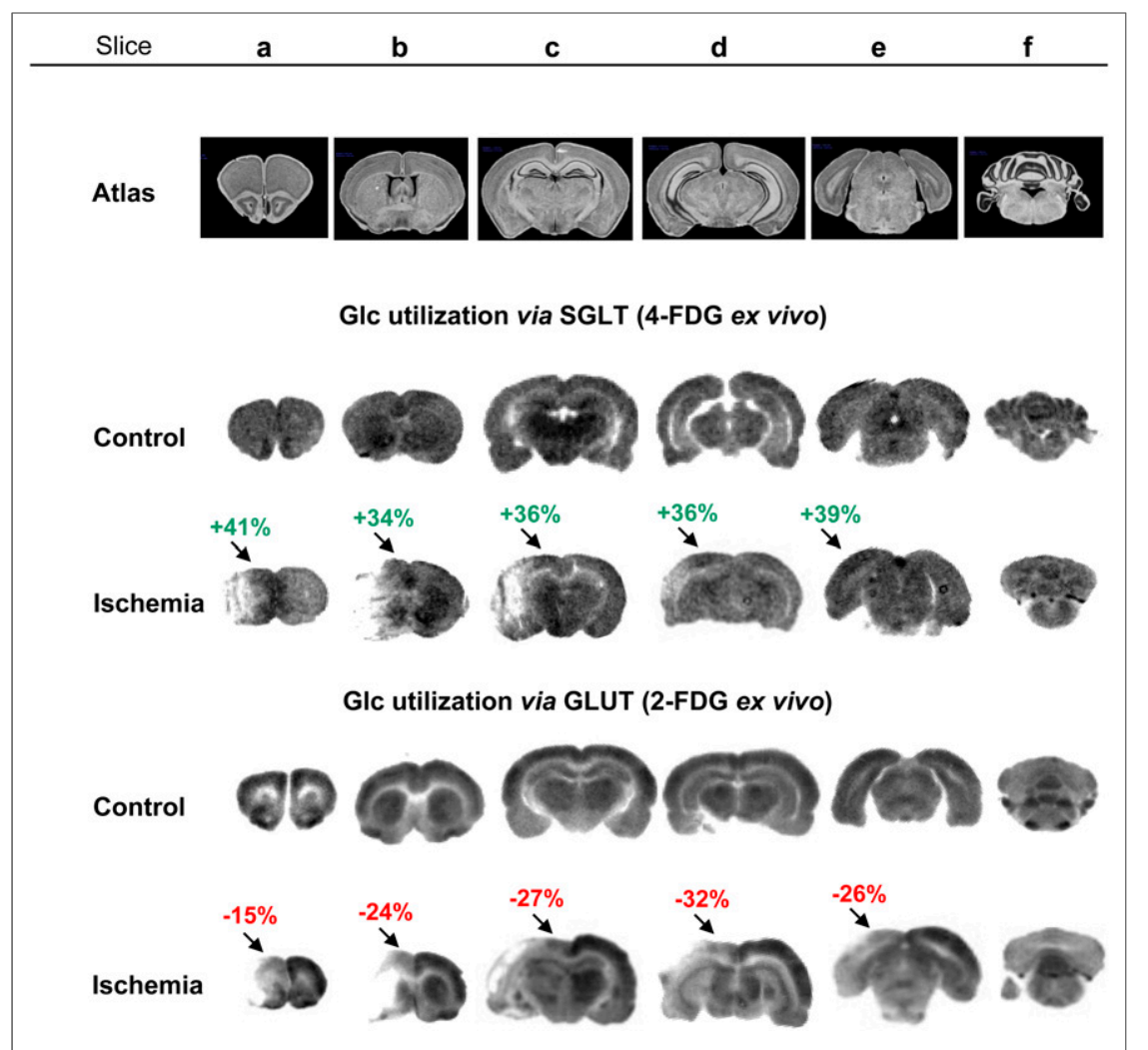

FIGURE 5. Upregulation of SGLT functional activity in rat model of middle-cerebral-artery occlusion (43). One hour after intravenous tail-veil injection of imaging probe (2-FDG or 4-FDG; 5-8 $\mathrm{mCi}$ ), ex vivo autoradiography was used to demonstrate different responses between SGLTs and GLUTs under postischemic conditions. One day after reperfusion, accumulation of both 4-FDG and 2-FDG was reduced in focal area, but in penumbra of focal lesions accumulation of 4-FDG was increased by $34 \%-41 \%$, in contrast to more than $15 \%$ reduction of $2-F D G$ uptake, as is consistent with previous work (44). Regions of interest for quantification of 4-FDG or 2-FDG were manually drawn on penumbral regions and same regions on contralateral side using Multi Gauge, version 3.0. Glc = glucose. (Courtesy of Amy S. Yu, Ernest M. Wright, Jorge R. Barrio.)

from SGLT- to GLUT-mediated glucose transport was recently demonstrated in human lung adenocarcinomas and in murine genetically engineered models with a combination of immunohistochemistry and Me-4FDG PET imaging (30). Premalignant and early-stage/low-grade lung adenocarcinoma expressed high functional activity of SGLT2; GLUT-mediated uptake of 2-FDG was observed mainly in more advanced stages of lung adenocarcinoma, suggesting a global shift from SGLT- to GLUT-mediated transport (Fig. 4). This result is consistent with the clinical role of 2-FDG PET in lung adenocarcinoma-high sensitivity and specificity in moderate and advanced stages but unreliability in earlier stages. Therefore, using 2-FDG PET to evaluate its uptake in lung cancer would drastically change with the stage of cancer development, whether it was evaluated by $\mathrm{MR}_{\mathrm{Glc}}$ or by SUV. This may be the best evidence to date that there is a discrepancy in glucose metabolic rate that is clinically significant and is not accounted for by a 2-FDG PET determination. It is likely that the same shift will be observed in other cancers, emphasizing the importance of SGLT transporters in limiting our interpretation of SUVs for 2-FDG PET.

If tissues have abundant access to circulating glucose via facilitated transporters (GLUTs), the critical question is why they need energetically expensive glucose via SGLTs. The well-recognized role of SGLTs in intestine and kidney is focused on recovery of glucose for metabolic use in the whole body, but locally in tissues this role does not apply. The expression of SGLTs is cellularly heterogeneous. The exquisite localization of SGLT-mediated glucose accumulation against an extracellular gradient (22-24) provides a higher level of glucose in cellular microenvironments that presumably is driven by mitochondrial demand for an accessible fuel. This mechanism may be an advantage in early cancer cell development and may explain SGLTs in premalignant lesions and early tumorigenesis, as well as metastasis initiation. In sharp contrast, GLUT-mediated uptake of 2-FDG appears low at early stages of cancer (Fig. 4), which parenthetically limits its clinical role in early detection. In later stages of cancer, upregulation of glycolysis results in increased consumption of 2-FDG, a nearly universal property of advanced primary and metastatic cancers. The persistent metabolism of glucose to lactate even under aerobic conditions (aerobic glycolysis) in cancer development (31) leads to microenvironmental acid-induced toxicity for surrounding normal cells, which may promote unconstrained proliferation and invasion (32).

\section{ROAD MAP FOR INVESTIGATING THE IMPACT OF SGLTS ON 2-FDG PET IMAGING}

Having argued that the expression and function of SGLTs in cancer deserves further attention by the nuclear medicine community and biologic researchers, the obvious question is how this should be accomplished. Although it might be tempting to invoke genetic approaches, including knockout models, this would likely lead to a necessary but long road to answering the impact, which is the clinically relevant question. A compelling case could also be made for a bedside-tobench translational approach. Measuring SUVs for 2-FDG PET will always be the easiest and most commonly applied metric for estimating cellular energy metabolism. However, some investigations may find changes in SUV that are inconsistent with clinical assessment of the course of disease, and this is the setting where the clinical observation should lead to more detailed assessment of the role of SGLTs and other variables that might change LC. This approach starts by identifying situations in which there is valid suspicion that energy metabolism is more involved than what 2-FDG PET results predict, thus incentivizing directed tissue analysis for even specific animal models to learn about differential regulation of GLUTs and SGLTs. Cancers with high levels of SGLT expression should be considered. In addition to early-stage lung adenocarcinoma, cancer in the prostate, kidney, and pancreas is a setting in which 2-FDG has found a limited role, but SGLTs are functionally expressed. Analysis of biopsy specimens of tumor and surrounding normal tissue will tell whether SGLTs become dysregulated as cancers develop-for example, with selective upregulation in early disease and subsequent downregulation. If they are, that would be 
sufficient information to recommend caution in interpreting 2-FDG SUVs and might foster imaging with Me-4FDG or 4-FDG, as appropriate. Also, these results might stimulate laboratory studies as to whether SGLTs have an early tumor-promotion role or a later tumor-suppression role. Answers to these mechanistic questions would have consequences in terms of tumor biology and development of new therapeutic targets. Current practice recommends a minimum $30 \%$ change in SUV adjusted for lean body mass in order to report a response to tumor therapy (33). With a better understanding of factors that impact $\mathrm{MR}_{\mathrm{Glc}}$ or SUV measurements, could this threshold be reduced, making the nuclear medicine study a more useful consult?

The relative effect of SGLT1 and SGLT2 functional expression in the normal human brain confirms that the 2-FDG LC is globally relatively stable in the brain, where most of the glucose utilization is via GLUTs, with only about $5.4 \%$ of the glucose being consumed via SGLTs, as measured with 4-FDG PET (Appendix A provides estimates of relative glucose utilization). However, locally the effects may be more significant: SGLTs are highly expressed in cortical hippocampal glutamatergic neurons and in Purkinje cells in cerebellum (Fig. 3) $(25,26)$, both of which are part of the neuronal circuit of cerebral vulnerability to brain hypoxia (34). Yet, only limited data exist as to the variations in cerebral glucose transport and use via GLUTs or SGLTs in related disease conditions. Figure 5 provides a hint as to their possible role in stroke.

In cancer tissues, the heterogeneous cellular composition and the presence of quantitatively significant SGLT expression are consistent with the unreliable value of the 2-FDG LC. Cancer staging adds an imponderable variable at the earliest stages of its development, where SGLT-mediated transport may be especially high, masking the 2-FDG PET signal until a more glycolytic stage of cancer metabolism develops. The same logic seems to apply to myocardial glucose utilization, which in early determinations appears high via SGLT in the young human heart and evolves into a more glycolytic form with age (Ernest M. Wright and Jorge R. Barrio, unpublished data, 2014).

The observation on glucose transport and use via GLUTs and SGLTs in cancer adds a twist to the observation that cancer cells may use other substrates in addition to glucose. Assumptions that some human cancers consume little glucose, based on low 2-FDG PET signal, need to be reevaluated and confirmed only after glucose utilization via SGLTs is considered. Using both 2-FDG and Me-4FDG (or 4-FDG in brain) in humans would help provide comprehensive insight into the very basic biochemical mechanism of glucose utilization via GLUT and SGLT transporters in health and disease stages.

\section{CONCLUSION}

An accurate value of the LC is essential for quantitative assessment of glucose metabolic rates from 2-FDG PET imaging. Calibration of 2-FDG PET results to precisely reflect the glucose utilization rate has been always understood as an essential prerequisite to attributing any alteration in 2-FDG tissue kinetics to glycolytic activity. The overarching conclusion from this report is that 2-FDG PET images, even when analyzed semiquantitatively using SUV approaches, are valuable in the clinic, but there is a need for a more complete understanding of factors that impact 2-FDG PET images. The present work summarizes evidence on the potential impact of previously unrecognized glucose utilization via SGLTs on the GLUT-mediated glucose utilization measured with 2-FDG. SGLT transporters are widely distributed and functionally active under normal physiologic conditions and respond to substantial upregulation during early disease. Thus, SGLT-mediated GLUTs may have a dynamic role in glucose utilization and a crucial effect on the 2-FDG LC, directly affecting quantitative glucose metabolism measurements in humans using 2-FDG PET.

More research is needed to comprehend the intricacies of the in vivo interplay between GLUT- and SGLT-mediated glucose transport in physiologic and pathophysiologic states throughout the human body. The initial observations reported here open a myriad of interesting basic questions about in vivo glucose utilization, well beyond the current use and limitations of the 2-FDG PET approach. The recent observation suggesting that the epidermal growth factor receptor regulates SGLT expression in cancer (35) adds a critical variable to the comprehensive understanding of in vivo glucose utilization for optimization of new therapies for cancer patients (36).

\section{DISCLOSURE}

Financial support from the National Institutes of Health (grants NIH R01 DK077133, P01AG025831, and P01CA042045) is acknowledged. Jorge R. Barrio also gratefully acknowledges the support of the Elizabeth and Thomas Plott Chair Endowment in Gerontology. Jorge R. Barrio reports coauthorship in an intellectual property patented by the University of California (28). No other potential conflict of interest relevant to this article was reported.

\section{ACKNOWLEDGMENTS}

We thank Dr. Ernest M. Wright (UCLA School of Medicine) for review and valuable comments on this article.

\section{KEY POINTS}

QUESTION: Does the 2-FDG PET signal fully represent the total glucose utilization in tissue?

PERTINENT FINDINGS: The GLUT-mediated in vivo glucose utilization measured by 2-FDG PET would be masked to the potentially significant role in glucose transport and use of functional SGLTs present in normal human tissues (e.g., brain, heart, and muscle) and cancer. The interplay between GLUT- and SGLTmediated glucose utilization would affect the 2-FDG PET signal, the LC, and the SUV used for quantitative in vivo measurement of glucose utilization.

IMPLICATIONS FOR PATIENT CARE: The apparent variations in 2-FDG PET signals have been especially significant in tumors, particularly at different stages of cancer development, potentially limiting in several instances the diagnostic value of the biomarker, as well as its relevance in monitoring treatments.

\section{APPENDIX A}

Estimation of the Relative SGLT-Mediated Versus GLUT-Mediated Glucose Utilization in Normal Human Brain

Although specific retention of 2-FDG in human brain occurs via BBB GLUT1 transporters and hexokinase-mediated phosphorylation in neuronal cells, the specific trapping and retention of 4-FDG is through SGLTs on the neuronal cell membrane. On the basis of the Michaelis-Menten constants of 4-FDG for SGLT and GLUT as compared with those of glucose, as shown in Table 1, the LC of 4-FDG can be estimated to be about 5 times larger than that of 2-FDG.

The central question is how the brain uptake of 4-FDG is related to the actual glucose utilization rate. Dynamic scanning of normal 
healthy subjects after bolus injection of 4-FDG has been obtained together with dynamic blood 4-FDG levels during the 60-min scan time. For comparison, a similar dynamic 2-FDG PET and dynamic blood curve was also performed on the same subjects. Patlak analyses were done for representative cortical regions (i.e., frontal cortex and cerebellum for both the 4-FDG and 2-FDG PET determinations), and the uptake constants $\left(K_{i}\right)$ so obtained were compared with estimated glucose utilization rates via SGLTs and GLUTs. The $K_{i}$ of 4-FDG in the frontal cortex was found to be $0.0108 \mathrm{~mL} / \mathrm{min} / \mathrm{g}$, whereas that of $2-\mathrm{FDG}$ was $0.0430 \mathrm{~mL} / \mathrm{min} / \mathrm{g}$. On the basis of the larger LC (5 times) of 4-FDG than of 2-FDG, the glucose utilization rate through SGLTs in the frontal cortex is calculated to be about $5.4 \%$ of the rate assessed by 2 -FDG. In other words, in the frontal cortex, the glucose utilization rate via SGLT is relatively small as compared with that measured using 2-FDG. However, since 4-FDG distribution is different from 2-FDG distribution, as shown in Figure 4, it should be expected that regional variations would exist. For example, the high expression of SGLTs in the Purkinje cells in cerebellum is shown by the high cerebellar 4-FDG uptake (the $K_{i}$ value for 4-FDG there was determined to be $0.017 \mathrm{~mL} / \mathrm{min} / \mathrm{g}$ in cerebellum vs. $0.0108 \mathrm{~mL} / \mathrm{min} / \mathrm{g}$ in cortex $)$. Thus, in normal cerebellum a larger fraction $(\sim 8.5 \%)$ of glucose utilization is through SGLTs.

\section{REFERENCES}

1. Barrio JR. The molecular basis of disease. In: Phelps ME, ed. PET: Molecular Imaging and Its Biological Applications. New York, NY: Springer-Verlag; 2004: 270-320.

2. Sokoloff L, Reivich M, Kennedy C, et al. The $\left[{ }^{14} \mathrm{C}\right]$ deoxyglucose method for the measurement of local cerebral glucose utilization: theory, procedure, and normal values in the conscious and anesthetized albino rat. J Neurochem. 1977;28:897-916.

3. Di Chiro G. Positron emission tomography using $\left[{ }^{18} \mathrm{~F}\right]$ fluorodeoxyglucose in brain tumors: a powerful diagnostic and prognostic tool. Invest Radiol. 1987;22:360-371.

4. Duelli R, Kuschinsky W. Brain glucose transporters: relationship to local energy demand. News Physiol Sci. 2001;16:71-76.

5. Reivich M, Kuhl D, Wolf A, et al. The $\left[{ }^{18} \mathrm{~F}\right]$ fluorodeoxyglucose method for the measurement of local cerebral glucose utilization in man. Circ Res. 1979;44: 127-137.

6. Phelps ME, Huang SC, Hoffman EJ, Selin C, Sokoloff L, Kuhl DE. Tomographic measurement of local cerebral glucose metabolic rate in humans with (F-18)2fluoro-2-deoxy-D-glucose: validation of method. Ann Neurol. 1979;6:371-388.

7. Reivich M, Alavi A, Wolf A, et al. Glucose metabolic rate kinetic model parameter determination in humans: the lumped constants and rate constants for $\left[{ }^{18} \mathrm{~F}\right]$ fluorodeoxyglucose and [ $\left.{ }^{[\mathrm{C}} \mathrm{C}\right]$ deoxyglucose. J Cereb Blood Flow Metab. 1985;5:179-192.

8. Graham MM, Muzi M, Spence A, et al. The FDG lumped constant in normal human brain. J Nucl Med. 2002;43:1157-1166.

9. Hasselbalch SG, Madsen PL, Knudsen GM, Holm S, Paulson OB. Calculation of the FDG lumped constant by simultaneous measurement of global glucose and FDG metabolism in humans. J Cereb Blood Flow Metab. 1998;18:154-160.

10. Wu HM, Bergsneider M, Glenn TC, et al. Measurement of the global lumped constant for 2-deoxy-2- $\left[{ }^{18} \mathrm{~F}\right]$ fluoro-D-glucose positron emission tomography imaging: a method with validation based on multiple methodologies. Mol Imaging Biol. 2003;5:32-41.

11. Lammertsma AA, Brooks DJ, Frackowiak RS, et al. Measurement of glucose utilization with $\left[{ }^{18} \mathrm{~F}\right] 2$-fluoro-2-deoxy-D-glucose: a comparison of different analytical methods. J Cereb Blood Flow Metab. 1987;7:161-172.

12. Spence AM, Muzi M, Graham MM, et al. Glucose metabolism in human malignant gliomas measured quantitatively with PET, $\left[1-{ }^{11} \mathrm{C}\right]$ glucose and FDG: analysis of the FDG lumped constant. J Nucl Med. 1998;39:440-448.

13. Wu HM, Bergsneider M, Zhou Y, et al. A method for measuring the global lumped constant of FDG in traumatic brain injury patients [abstract]. $\mathrm{J} \mathrm{Nucl} \mathrm{Med}$. 2000;41(suppl):62P.

14. Wu HM, Huang SC, Hattori N, et al. Selective metabolic reduction in gray matter acutely following human traumatic brain injury. J Neurotrauma. 2004;21:149-161.

15. Krivokapich J, Huang SC, Selin C, Phelps ME. Fluorodeoxyglucose rate constants, lumped constants, and Fick metabolic rate under varied conditions in rabbit myocardium. Am J Physiol. 1987;252:H777-H787.
16. Williams KV, Price JC, Kelley DE. Interactions of impaired glucose transport and phosphorylation in skeletal muscle insulin resistance: a dose-response assessment using positron emission tomography. Diabetes. 2001;50:2069-2079.

17. Vertanen KA, Peltoniemi P, Marjamäki $\mathrm{P}$, et al. Human adipose tissue glucose uptake determined using $\left[{ }^{18} \mathrm{~F}\right]$-fluoro-deoxy-glucose $\left(\left[{ }^{18} \mathrm{~F}\right] \mathrm{FDG}\right.$ and PET in combination with microdialysis. Diabetologia. 2001;444:2171-2179.

18. Iozzo P, Jarvisalo MJ, Kiss J, et al. Quantification of liver glucose metabolism by positron emission tomography: validation study in pigs. Gastroenterology. 2007;132: 531-542.

19. Muzi M, Freeman SD, Burrows RC, et al. Kinetic characterization of hexokinase isoenzymes from glioma cells: implications for FDG imaging in human brain tumors. Nucl Med Biol. 2001;28:107-116.

20. Spence AM, Muzi M, Graham MM, et al. FDG and glucose uptake in malignant gliomas before and after radiotherapy: correlation with outcome. Clin Cancer Res. 2002;8:971-979.

21. Burrows RC, Freeman SD, Charlop AW, et al. $\left[{ }^{18} \mathrm{~F}\right]$-2-fluoro-2-deoxyglucose transport kinetics as a function of extracellular glucose concentration in malignant glioma, fibroblast and macrophage cells in vitro. Nucl Med Biol. 2004;31:1-9.

22. Díez-Sampedro A, Wright EM, Hirayama BA. Residue 457 controls sugar binding and transport in the $\mathrm{Na}(+) /$ glucose cotransporter. J Biol Chem. 2001;276: 49188-49194.

23. Wright EM, Loo DD, Hirayama BA. Biology of human sodium glucose transporters. Physiol Rev. 2011;91:733-794.

24. Wright EM. Glucose transport families SLC5 and SLC50. Mol Aspects Med. 2013;34:183-196.

25. Yu AS, Hirayama BA, Timbol G, et al. Functional expression of SGLTs in rat brain. Am J Physiol Cell Physiol. 2010;299:C1277-C1284.

26. Yu AS, Hirayama BA, Timbol G, et al. Regional distribution of SGLT activity in rat brain in vivo. Am J Physiol Cell Physiol. 2013;304:C240-C247.

27. Scafoglio C, Hirayama B, Kepe V, et al. Functional expression of sodium-glucose transporters in cancer. Proc Natl Acad Sci USA. 2015;112:E4111-E4119.

28. Wright EM, Barrio JR, Hirayama BA, Kepe V, inventors; University of California, assignee. Tracers for monitoring the activity of sodium/glucose cotransporters in health and disease. U.S. patent 8845999B2. September 30, 2014.

29. Kepe V, Scafoglio C, Liu J, et al. A positron emission tomography tracer targeting a sodium glucose cotransporter provides enhanced imaging of high grade astrocytomas and new pathobiology insights. J Neurooncol. 2018;138:557-569.

30. Scafoglio CR, Villegas B, Abdelhady G, et al. Sodium-glucose transporter 2 is a diagnostic and therapeutic target for early-stage lung adenocarcinoma. Sci Transl Med. 2018;10:eaat5933.

31. Lunt SY, Vander Heiden MG. Aerobic glycolysis: meeting the metabolic requirements of cell proliferation. Annu Rev Cell Dev Biol. 2011;27:441-464.

32. Gatenby RA, Gillies RJ. Why do cancers have high aerobic glycolysis? Nat Rev Cancer. 2004;4:891-899.

33. Wahl RL, Jacene H, Kasamon Y, Lodge MA. From RECIST to PERCIST: evolving considerations for PET response criteria in solid tumors. $\mathrm{J}$ Nucl Med. 2009;50(suppl 1):122S-150S.

34. Cervós-Navarro J, Diemer NH. Selective vulnerability in brain hypoxia. Crit Rev Neurobiol. 1991;6:149-182.

35. Engelman JA, Cantley LC. A sweet new role for EGFR in cancer. Cancer Cell. 2008; 13:375-376

36. Shankar LK, Hoffman JM, Bacharach S, et al. Consensus recommendations for the use of ${ }^{18} \mathrm{~F}$-FDG PET as an indicator of therapeutic response in patients in National Cancer Institute Trials. J Nucl Med. 2006;47:1059-1066.

37. Loo DD, Hirayama BA, Sala-Rabanal M, Wright EM. How drugs interact with transporters: SGLT1 as a model. J Membr Biol. 2008;223:87-106.

38. Parent L, Wright EM. Electrophysiology of the $\mathrm{Na}^{+}$/glucose cotransporter. In: Russell RL Jr, Jennings ML, eds. Molecular Biology and Function of Carrier Proteins. New York, NY: Rockefeller University Press; 1993:254-281.

39. Barnett JE, Holman GD, Munday KA. Structural requirements for binding to the sugar-transport system of the human erythrocyte. Biochem J. 1973;131:211-221.

40. Brahm J. Kinetics of glucose transport in human erythrocytes. J Physiol (Lond). 1983;339:339-354.

41. Bessell EM, Foster AB, Westwood JH. The use of deoxyfluoro-D-glucopyranoses and related compounds in a study of yeast hexokinase specificity. Biochem J. 1972; 128:199-204.

42. Sols A, Crane RK. Substrate specificity of brain hexokinase. J Biol Chem. 1954; 210:581-595.

43. Wang-Fischer Y, Koetzner L. Microsurgical instruments for stroke studies. In: Wang-Fischer Y, ed. Manual of Stroke Models in Rats. Boca Raton, FL: CRC Press; 2009:81-92.

44. Liu Y, Belayev L, Zhao W, et al. The effect of bone morphogenetic protein-7 (BMP-7) on functional recovery, local cerebral glucose utilization and blood flow after transient focal cerebral ischemia in rats. Brain Res. 2001;905:81-90. 